\title{
Astragaloside IV alleviates myocardial damage induced by type 2 diabetes via improving energy metabolism
}

\author{
ZHEN ZHANG $^{1}$, JING WANG ${ }^{2}$, YINGWEI ZHU ${ }^{3}$, HUI ZHANG ${ }^{1}$ and HONGXIN WANG ${ }^{1}$ \\ ${ }^{1}$ Key Laboratory of Cardiovascular and Cerebrovascular Drug Research of Liaoning Province, Jinzhou Medical University; \\ ${ }^{2}$ The First Affiliated Hospital, Jinzhou Medical University, Jinzhou, Liaoning 121001; \\ ${ }^{3}$ Institute of Physical Education, Bohai University, Jinzhou, Liaoning 121013, P.R. China
}

Received February 26, 2019; Accepted July 30, 2019

DOI: $10.3892 / \mathrm{mmr} .2019 .10716$

\begin{abstract}
The aim of the present study was to evaluate the protective effect and mechanism of Astragaloside IV (ASIV) on myocardial injury induced by type 2 diabetes, with a focus on energy metabolism. Blood glucose, the hemodynamic index, left ventricular weight/heart weight (LVW/HW), the left ventricular systolic pressure (LVSP), the left ventricular end diastolic pressure (LVEDP) and cell survival rate were measured in streptozotocin-induced diabetes model rats. Western blot analysis, PCR, hematoxylin-eosin and TUNEL staining, flow cytometry and ELISA were used to detect: i) Cardiomyocyte damage indicators such as atrial natriuretic peptide (ANP), brain natriuretic peptide (BNP), cytochrome $c$ (Cyt C), caspase-3, cleaved caspase-3 and the apoptotic rate; ii) energy metabolism indicators such as ATP/AMP and ADP/AMP; and iii) energy metabolism associated pathway proteins such as peroxisome proliferator-activated receptor $\gamma$ coactivator $1-\alpha$ (PGC-1 $\alpha$ ) and nuclear respiratory factor 1 (NRF1). The present demonstrated increased blood glucose, LVW/HW, LVSP, LVEDP and the cardiomyocyte damage indicators (ANP, BNP, Cyt C and caspase-3), in the diabetic and high glucose-treated groups, which were decreased by ASIV. The expression of NRF-1 and PGC-1 $\alpha$ significantly changed in the model group and was markedly improved following ASIV treatment. Furthermore, the abnormal energy metabolism in the model group was reversed by ASIV. According to the results, ASIV can regulate energy metabolism by regulating the release of PGC-1 $\alpha$ and NRF1 to rescue the abnormal energy
\end{abstract}

Correspondence to: Professor Hongxin Wang, Key Laboratory of Cardiovascular and Cerebrovascular Drug Research of Liaoning Province, Jinzhou Medical University, 40 (Section 3) Songpo Road, Linghe, Jinzhou, Liaoning 121001, P.R. China

E-mail: hongxinwang@jzmu.edu.cn

Key words: diabetic cardiomyopathy, astragaloside IV, apoptosis, hypertrophy, mitochondrial energy metabolism, peroxisome proliferator-activated receptor $\gamma$ coactivator $1-\alpha$, nuclear respiratory factor 1 metabolism caused by diabetes mellitus, thus decreasing the myocardial damage caused by diabetic cardiomyopathy.

\section{Introduction}

Diabetes is a metabolic disease, with the number of people with diabetes estimated to reach 700 million worldwide in 2040 (1). Cardiovascular complications are the main cause of mortality in diabetic patients, and $\sim 80 \%$ of diabetic patients die from cardiovascular diseases (2). Diabetic cardiomyopathy (DCM) has the highest mortality rate associated with diabetic complications, and the methods for treatment are poor (3). In the early stage of the disease, patients exhibit symptoms such as ventricular diastolic dysfunction and myocardial remodeling; as the disease worsens, microvascular disease and the necrosis of myocardial tissue occurs, eventually leading to heart failure (4).

Although there are many unknown mechanisms of in diabetic cardiomyopathy, previous studies have shown that metabolic disorders have a significant role in the pathogenesis of diabetic cardiomyopathy. As the body does not normally use glucose to supply energy, the oxidation of fatty acids is exacerbated, causing toxicity from sugar and fat. This damages the mitochondria in myocardial cells (5), inducing mitochondrial dysfunction and deficits in ATP production, and the damage can eventually lead to the apoptosis of myocardial cells, hypertrophy and fibrosis, and, ultimately, cardiac dysfunction (6). Peroxisome proliferator-activated receptor $\gamma$ coactivator $1 \alpha$ (PGC-1 $\alpha$ ), which is one of the members of the PGC-1 $\alpha$ family, plays a vital role in the process of biosynthesis in mitochondria (7-9); it can stimulate nuclear transcription factor nuclear respiratory factor (NRF) and other downstream factors to promote the proliferation of mitochondria and the expression of proteins in mitochondria, regulate cellular energy metabolism and provides ATP to heart tissue (10). The increased expression of PGC-1 $\alpha$ can improve myocardial metabolic disorders (11).

Following stimulation by adverse environmental factors, the apoptosis of cardiac cells is increased, and changes in cell function and structure lead to cardiac hypertrophy. Under conditions of high glucose, a series of metabolites generated by cardiac cells can stimulate the binding of extracellular apoptosis-associated ligands to specific receptors on the cell 
membrane, activating cysteine-containing aspartate-specific proteases (caspases), triggering a series of apoptosis pathways and accelerating the process of apoptosis (12). The abnormal structure or function of mitochondria can lead to abnormal energy metabolism, which not only damages myocardial function but also causes inflammation and oxidative stress to further aggravate the damage. Therefore, restoring mitochondrial function is key to the treatment of diabetic myocardium (13).

Astragaloside IV (ASIV) is a saponin compound isolated from Astragalus that has the anti-apoptotic, anti-oxidative and glucose-controlling effects; therefore it has a certain therapeutic effect on diabetic cardiomyopathy (14). However, the pharmacological action of ASIV on diabetic cardiomyopathy is still unclear and requires further investigation. Previous studies have found that ASIV can improve energy metabolism dysfunction induced by isoproterenol in rats by increasing the expression of PGC-1 $\alpha$ by isoproterene in rats (15-20).

The aim of the present study was to investigate the pharmacological mechanism of ASIV in diabetic cardiomyopathy by focusing on the aspects of energy metabolism and PGC-1 $\alpha$.

\section{Materials and methods}

Reagents. ASIV was purchased from Nanjing Jingzhu Bio-Technology Co., Ltd. Streptozotocin (STZ) and carboxymethyl cellulose sodium (CMC-Na) were purchased from Sigma-Aldrich (Merck KGaA). A TUNEL kit (In Situ Cell Death Detection kit, AP) was purchased from Roche Molecular Diagnostics. ATP (kt39623), ADP (kt210319) and AMP (kt28319) ELISA kits were purchased from MSKBIO Co. Ltd. A BCA Protein Assay kit was purchased from Beyotime Institute of Biotechnology. TRIzol reagent and a reverse transcription-PCR (RT-PCR) kit were purchased from Dingguo Biological Co. Ltd. PGC-1 $\alpha$, NRF1, atrial natriuretic peptide (ANP) and brain natriuretic peptide (BNP) were purchased from ABclonal. Cleaved caspase-3, caspase-3 and cytochrome $c$ (Cyt C) were purchased from Biological Technology Co. Ltd.

Animals and experimental design. Healthy male Sprague-Dawley rats (6-8 weeks old, 180-200 g, $n=50$ ) were purchased from the Experimental Animal Center of Jinzhou Medical University (Jinzhou, China). All experiments and procedures were approved by the Medical Ethics Committee of Jinzhou Medical University (approval no. LNMU-2016-121). The rats were treated in accordance with the Guide for the Care and Use of Laboratory Animals (8th edition, National Academies Press) (21). The rats were adapted to their new environment (at a temperature of $20-23^{\circ} \mathrm{C}$, humidity from $30-48 \%$, and a 12-h light/dark cycle) for 1 week before the experiment. There were 5 groups in the in vivo experiments, and each group consisted of 10 rats. Healthy male SD rats $(n=40)$ were injected with STZ through the tail vein at a dose of $35 \mathrm{mg} / \mathrm{kg}$. The fasting blood glucose level was detected 1 week later. If an animal presented with a fasting blood glucose level $>16.7 \mathrm{mM}$ and symptoms of polydipsia, polyuria and polyphagia, it was considered a diabetic model rat. Diabetes was successfully established in 40 rats and 30 of them were randomly selected and randomly divided into three groups of 10 each. The ASIV-high (H), ASIV-mid (M) and ASIV-low (L) groups were established by the intraperitoneal injection of three different doses of ASIV (40, 20 and $10 \mathrm{mg} / \mathrm{kg}$, respectively) once a day. ASIV was dissolved in $1 \% \mathrm{CMC}$. The remaining 10 rats were used for the diabetic model only group, and $10 \mathrm{SD}$ rats were used as the control group. The same volume of $1 \% \mathrm{CMC}$ was administered daily. Blood glucose was measured and recorded on day 1, and weeks 2, 4, 8, 12 and 16 following administration. After 16 weeks of ASIV treatment, rats were anesthetized [urethane, $1.5 \mathrm{~g} / \mathrm{kg}$, intraperitoneal injection (i.p.)] (22), and hemodynamic parameters were measured. Rats were euthanized by cervical dislocation under anesthesia (diazepam, $10 \mathrm{mg} / \mathrm{kg}$ and ketamine, $50 \mathrm{mg} / \mathrm{kg}$ ) and myocardial tissue was collected. Some of the tissues were fixed with $4 \%$ paraformaldehyde solution at room temperature for $24 \mathrm{~h}$, and the remaining tissue was stored at $-86^{\circ} \mathrm{C}$.

Cell culture. H9C2 cells were purchased from Wuhan Boster Biotech Company, Ltd. and the cells were propagated in DMEM (Gibco; Thermo Fisher Scientific, Inc.) supplemented with 10\% FBS (HyClone; GE Healthcare Life Sciences) and $1 \%$ penicillin-streptomycin (Invitrogen; Thermo Fisher Scientific, Inc.). The H9C2 cells were divided into six groups: Con (control), mannitol (control + mannitol $7.5 \mathrm{mM}$ ), high glucose (HG; glucose $33.3 \mathrm{mmol}^{-1} \mathrm{l}^{-1}$, $\mathrm{HG}+\mathrm{ASIV}-\mathrm{L}$ $\left(20 \mu \mathrm{mol} \cdot \mathrm{l}^{-1}\right), \mathrm{HG}+\mathrm{ASIV}-\mathrm{M}\left(40 \mu \mathrm{mol} \cdot \mathrm{l}^{-1}\right)$ and $\mathrm{HG}+\mathrm{ASIV}-\mathrm{H}$ $\left(80 \mu \mathrm{mol} \cdot 1^{-1}\right)$.

Detection of cardiac function indicators. After being anesthetized with $20 \%$ urethane $(1.5 \mathrm{~g} / \mathrm{kg}$, i.p.), all rats were weighed before the beginning of the experiment. A physiological recorder was used to monitor the left ventricular systolic pressure (LVSP), the left ventricular end diastolic pressure (LVEDP) and the maximum left ventricular ascending/descending rate $\left( \pm \mathrm{dp} / \mathrm{dt}_{\max }\right)$, after the right common carotid artery was isolated and intubated into the left ventricle. The hearts of the rats were harvested and rinsed with precooled normal saline, and then dissected and weighed. The ratio of the left ventricle weight to the heart weight ( $\mathrm{LVW} / \mathrm{HW}$ ) was separately calculated. Finally, the heart tissues retained for slicing were placed in $4 \%$ polyformaldehyde and the remaining heart tissues were stored at $-86^{\circ} \mathrm{C}$.

Hematoxylin and eosin $(H \& E)$ and TUNEL staining. After soaking in $4 \%$ formaldehyde at room temperature for $24 \mathrm{~h}$, the heart tissues were embedded in paraffin and sectioned into $5-\mu \mathrm{m}$ sections. The sections were stained using H\&E (hematoxylin 2-3 min and eosin $1 \mathrm{~min}$, both at room temperature) and TUNEL kits [50 $\mu 1$ TdT and dUTP (1:9) in a dark moist chamber at $37^{\circ} \mathrm{C}$ for $1 \mathrm{~h}$, respectively. An inverted microscope (DMI3000B, Leica Microsystems GmbH) was used for observation (magnification, $\mathrm{x} 400$ ), and the matching software LAS v4.3 was used to capture and analyze images. The apoptotic index (\%) was calculated as the number of positive apoptotic cardiomyocytes/the total number of cardiomyocytes $\mathrm{x} 100$.

Detection of the cell survival by MTT. H9C2 cells (Cell concentration $\sim 5 \times 10^{4} / \mathrm{ml}$ ) were seeded onto 96 -well plates ( 8 wells per group) and cultured at $37^{\circ} \mathrm{C}$ and $5 \% \mathrm{CO}_{2}$ for $24 \mathrm{~h}$. After $48 \mathrm{~h}$, $20 \mu \mathrm{l}$ MTT ( $5 \mathrm{mg} / \mathrm{ml})$ was added, and the cells were incubated 
for $4 \mathrm{~h}$. Subsequently, $150 \mu \mathrm{l}$ DMSO was added, and the plates were shaken for $10 \mathrm{~min}$ to dissolve the formazan crystals. The absorbance value (OD value) was measured at a wavelength of $490 \mathrm{~nm}$ using an enzyme-labeling instrument. Cell viability was calculated.

Apoptosis assays. Apoptosis was detected using an Annexin V-FITC apoptosis detection kit (Annexin V-FITC/PI Apoptosis Detection kit, Dalian Meilun Biology Technology Co., Ltd.) according to the manufacturer's instructions. The cells in each group $\left(5 \times 10^{5-6}\right.$ cells $\left./ \mathrm{ml}\right)$ were extracted, $100 \mu 1$ $1 \mathrm{X}$ binding buffer was added, and the cells were resuspended gently. After adding $5 \mu \mathrm{l}$ Annexin V-FITC and $5 \mu \mathrm{l}$ propidium iodide, the cells were mixed gently and incubated at room temperature without light for $10 \mathrm{~min}$. Following the addition of $400 \mu \mathrm{l} 1 \mathrm{X}$ binding buffer and suspending the cells gently, Annexin V-FITC staining was detected using a BD Accuri C6 Plus flow cytometer (BD Biosciences) and analyzed by FlowJo v10 (FlowJo LLC).

Detection of ATP/ADP and ATP/AMP. According to the ELISA kit instructions, the OD value was detected and recorded. First, standard curves were generated, and the related reagents were added to the myocardial tissues and $\mathrm{H} 9 \mathrm{C} 2$ cells. Then, ATP, $\mathrm{ADP}$ and AMP were calculated by measuring the OD values and the standard curves of each group. Finally, the ATP/ADP and ATP/AMP ratio were obtained.

Preparation of protein extracts and western blot analysis. Nucleoproteins and mitochondrial proteins were extracted from the myocardial tissue and $\mathrm{H} 9 \mathrm{C} 2$ cells with corresponding kits (Tissue or Cell Total Protein Extraction kit, Sangon Biotech Co. Ltd.; Nuclear Protein Extraction kit, Beijing Solarbio Science \& Technology Co., Ltd.; Tissue Mitochondria Isolation kit, Novland Biopharma Co. Ltd.; www.novland. com.cn) The procedure was performed according to the manufacturer's instructions. The total protein, the cytoplasmic protein and the cytoplasmic protein after the removal of mitochondria were extracted. After the protein concentration was determined using the BCA method, the samples were prepared according to the required concentration and dosage ( $40 \mu \mathrm{g}$ of total protein to each lane). SDS-PAGE (10-12\% polyacrylamide gels) was used to separate protein samples. The isolated proteins were transferred to PVDF membranes (EMD Millipore) and blocked with 1\% BSA at room temperature for $2 \mathrm{~h}$. The membranes were incubated with primary antibodies against ANP (NPPA polyclonal antibody, ABclonal Biotech Co., Ltd. A14755, 1:1,000), BNP (BNP polyclonal antibody, ABclonal Biotech Co., Ltd. A2179, 1:1,000), Cyt C (anti-cytochrome $c$ antibody, Boster Biological Technology, A03529, 1:1,000), caspase-3 (anti-caspase-3 antibody, Boster Biological Technology PB9188, 1:1,000), cleaved caspase-3 (anti-Caspase-3 p12 antibody, Boster Biological Technology BM4620, 1:1,000), NRF1 (NRF1 polyclonal antibody, ABclonal Biotech Co., Ltd. A5547, 1:1,000), PGC-1 $\alpha$ (PGC1 $\alpha$ polyclonal antibody, ABclonal Biotech Co., Ltd. A12348, $1: 1,000)$ and $\beta$-actin ( $\beta$-actin Antibody, ProteinTech Group, Inc 66009-1-Ig, 1:20,000) at room temperature for $1.5 \mathrm{~h}$. Then the PVDF membrane was placed in the corresponding antibody (HRP-conjugated Affinipure Goat Anti-Rabbit

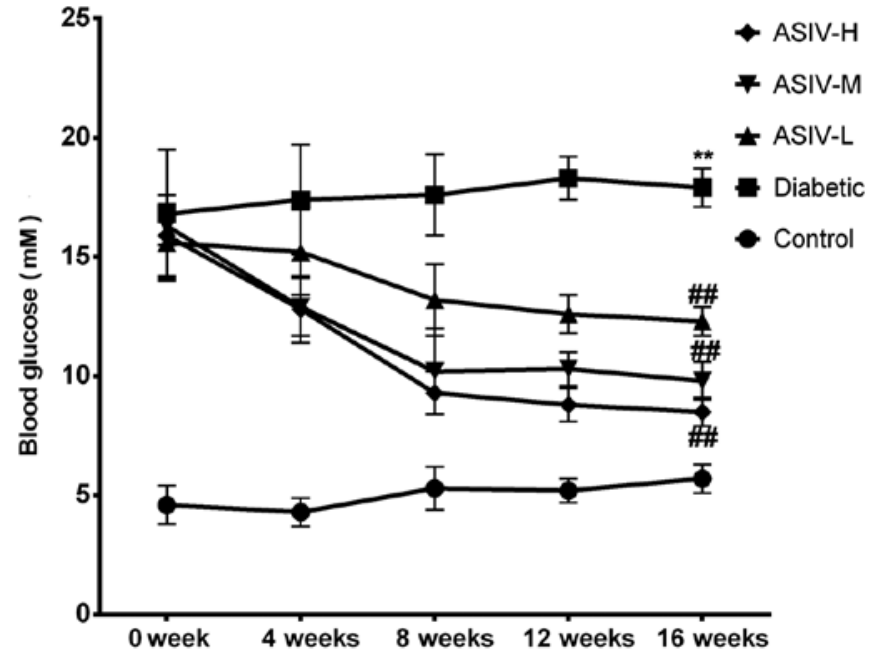

Figure 1. ASIV inhibits the elevation of blood glucose in STZ-induced diabetic rats. Data are expressed as the means $\pm \mathrm{SD}, \mathrm{n}=10,{ }^{* * *} \mathrm{P}<0.01$ vs. control group. ${ }^{\# \#} \mathrm{P}<0.01$ vs. diabetic group. ASIV, astragaloside IV.

$\operatorname{IgG}(\mathrm{H}+\mathrm{L})$, ProteinTech Group, Inc. SA00001-2, 1:20,000; HRP goat anti-mouse IgG (H+L), ABclonal Biotech Co., Ltd. AS003, 1:10,000) and incubated at room temperature for $2 \mathrm{~h}$. All antibodies were diluted with $0.1 \%$ BSA. The results were detected with enhanced chemiluminescence reagents (Wanleibio Co., Ltd.) and analyzed using Results Quantity One software v6.0.1 (Bio-Rad Laboratories, Inc.).

Statistical analysis. All data are expressed as the mean \pm SD and were analyzed using SPSS 19.0 software (IBM Corp.). Statistical analysis was performed using one-way ANOVA followed by Bonferroni's test. $\mathrm{P}<0.05$ was considered to indicate a statistically significant difference.

\section{Results}

ASIV has a regulatory effect on blood glucose in diabetic rats. A broken line graph (Fig. 1) of the blood glucose levels was made to observe the effect of ASIV on blood glucose. No significant changes in blood glucose level in the control group and the diabetic group over the 16-week experimental period, indicating that the solvent did not regulate blood glucose. The blood glucose of the three groups administered ASIV decreased markedly as the duration of ASIV administration increased, and the degree of the decrease was associated with the administered dose, indicating that ASIV has an effect on hypoglycemia.

ASIV improves the physiological indexes in STZ-induced diabetic rats and decreases the influence on tissue structure. Compared with the control group, the LVSP and $+\mathrm{dp}^{\mathrm{d}} \mathrm{dt}_{\max }$ in the diabetes model group were significantly decreased, indicating decreased myocardial systolic ability, whereas LVEDP was increased and $-\mathrm{dp} / \mathrm{dt}_{\max }$ decreased, reflecting decreased myocardial diastolic ability. Combined with the above signs of diabetic cardiomyopathy, myocardial diastolic function was abnormal and heart failure occurred. According to the results, the LVEDP gradually decreased as the dose increased, LVSP, $+\mathrm{dp} / \mathrm{dt}_{\max }$ and $-\mathrm{dp} / \mathrm{dt}_{\max }$ gradually increased, and myocardial 

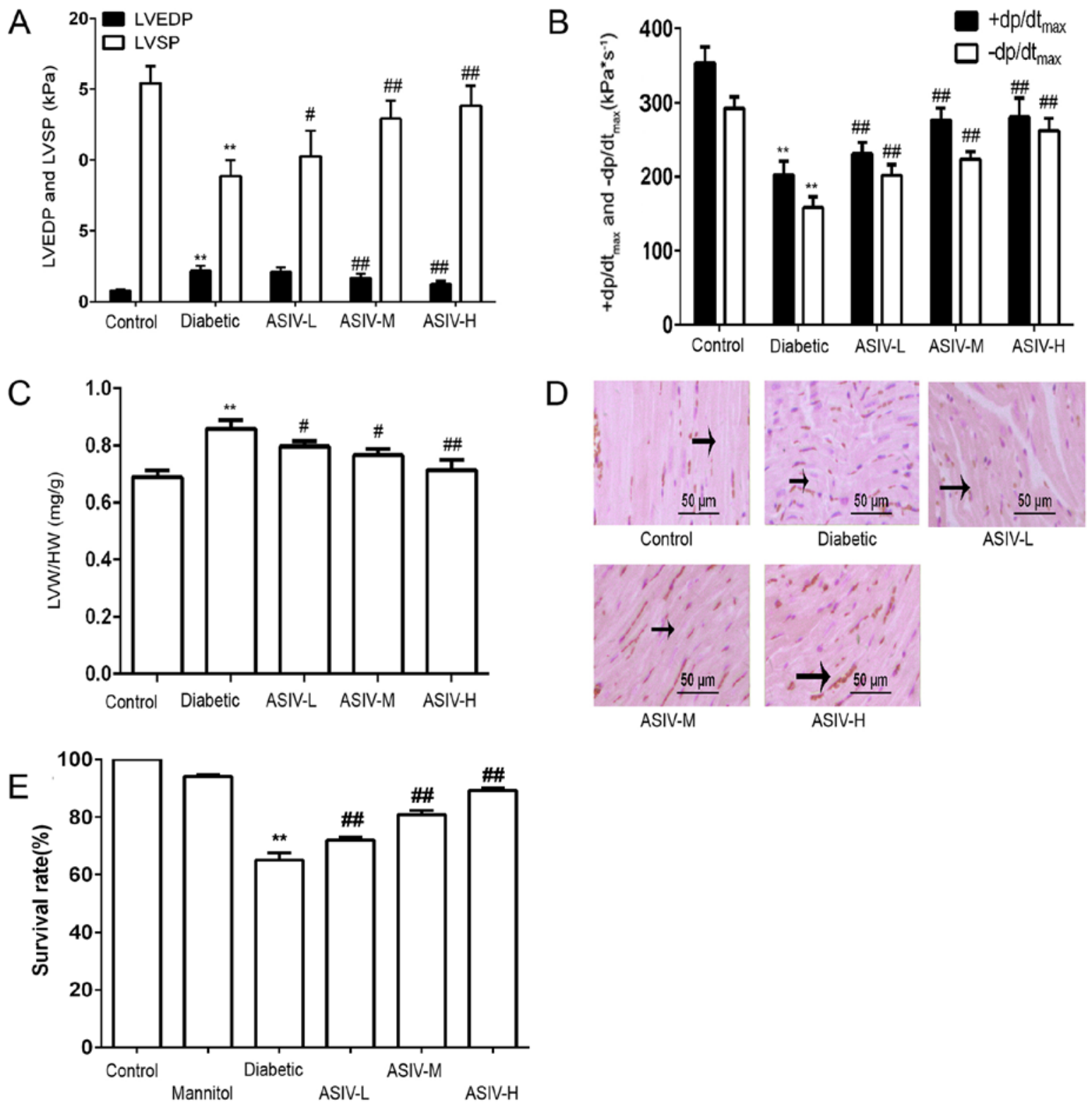

Figure 2. ASIV improves the physiological indexes of STZ-induced diabetic rats and decreases the effects of diabetes on tissue structures. (A) LVEDP and LVSP, (B) $\pm d p / \mathrm{dt}_{\max }$ and (C) $\mathrm{LVW} / \mathrm{HW}$ of rats in the different experimental groups. $\mathrm{n}=10$. (D) Hematoxylin and eosin staining in cardiac tissue sections of each group. The black arrow indicates myocardial fibers. (E) Cell survival of $\mathrm{H} 9 \mathrm{C} 2$ cells determined by MTT assay. $\mathrm{n}=8$ Data are expressed as the mean $\pm \mathrm{SD}$. ${ }^{* *} \mathrm{P}<0.01$ vs. the control group. ${ }^{\#} \mathrm{P}<0.05$ and ${ }^{\# \#} \mathrm{P}<0.01$ vs. diabetic group. LVEDP, left ventricular systolic pressure; LVSP, left ventricular end diastolic pressure; ASIV, astragaloside IV; L, low; M, mid; H, high; $\pm \mathrm{dp} / \mathrm{dt}_{\max }$, maximum left ventricular ascending/descending rate; LVW/HW, ratio of left ventricular weight to heart weight.

function was significantly improved by ASIV intervention (Fig. 2A and B). As shown in Fig. 2C, the LVW/HW was significantly increased in the diabetic group compared with the control animals, indicating that diabetes caused the left heart to increase in weight. Following the administration of ASIV, the LVW/HW decreased compared with the diabetic model group, and the degree of the decrease dependent on the ASIV concentration, demonstrating the effect of ASIV on the degree of myocardial hypertrophy.

$\mathrm{H} \& \mathrm{E}$ staining showed that the myocardial fibers in the heart tissues of diabetic rats were disordered and transversely broken. This damage was improved in the ASIV-treated groups. The therapeutic effect depended on the dose (Fig. 2D). The results of the cell viability assay (Fig. 2E) showed that cells survival in the control and mannitol groups was $>95 \%$. The survival rate in the HG group was significantly decreased compared to the control group. Furthermore, the survival rate increased following ASIV intervention. This demonstrated that ASIV can protect cells and alleviate the damage under high-glucose conditions.

ASIV decreases the damage of myocardial tissue damage in diabetic rats and $\mathrm{H} 9 \mathrm{C} 2$ cells in high-glucose conditions. As shown in Fig. 3A-D, the expression of ANP and BNP in the left ventricle of the diabetic group was significantly higher than that in the ventricles of the control group, indicating that the diabetic group exhibited myocardial hypertrophy. The expression of ANP and BNP decreased in the ASIV group. 
A

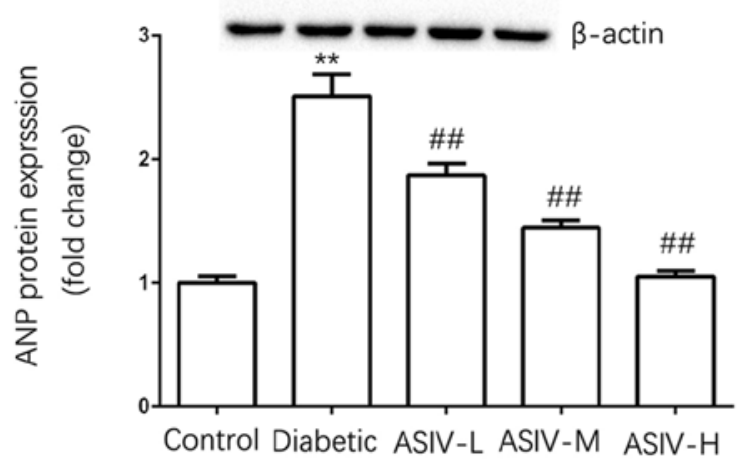

C

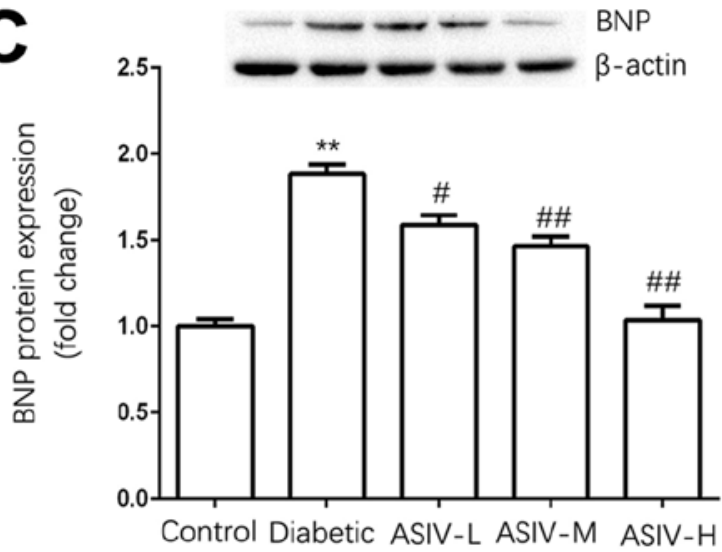

E

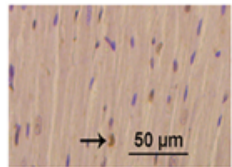

Control

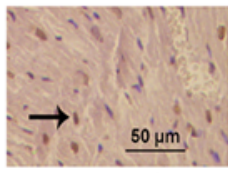

ASIV-M

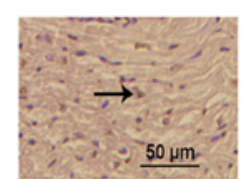

Diabetic

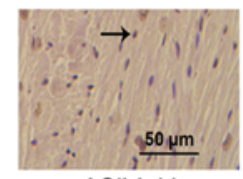

ASIV-H

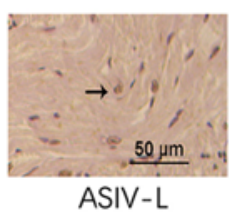

G
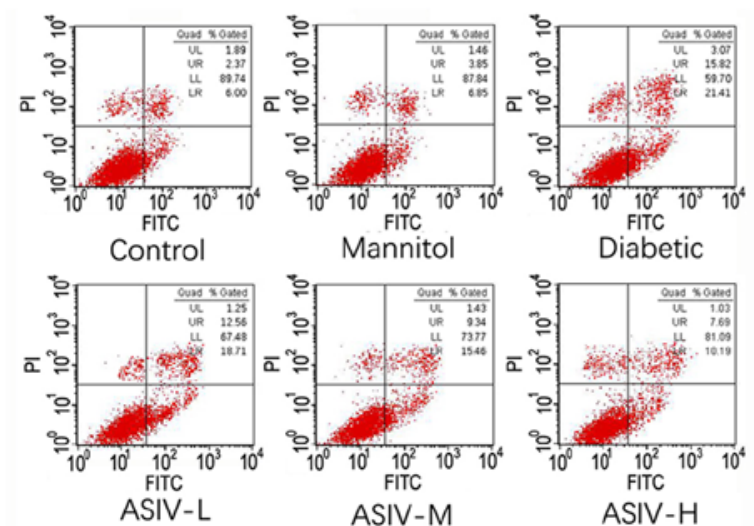

$\mathbf{F}$

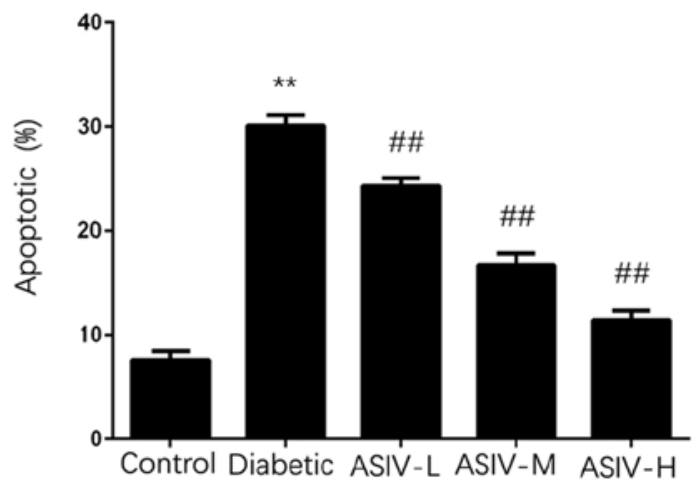

B

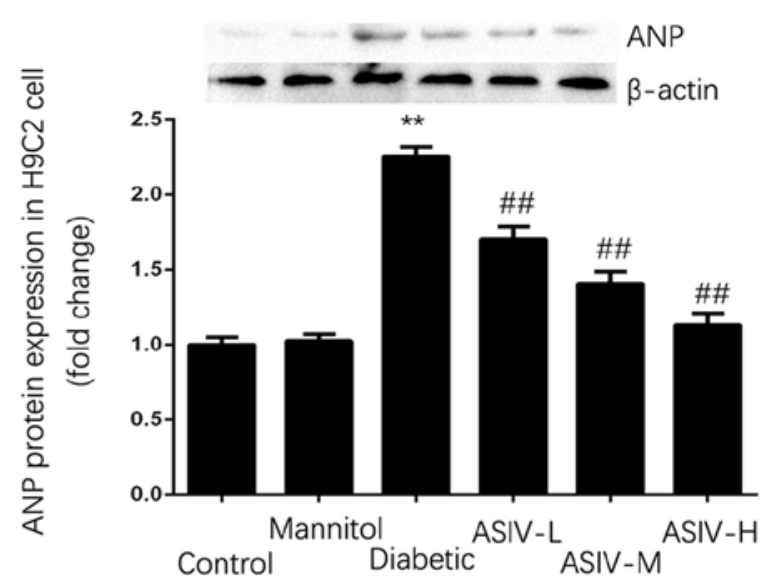

D

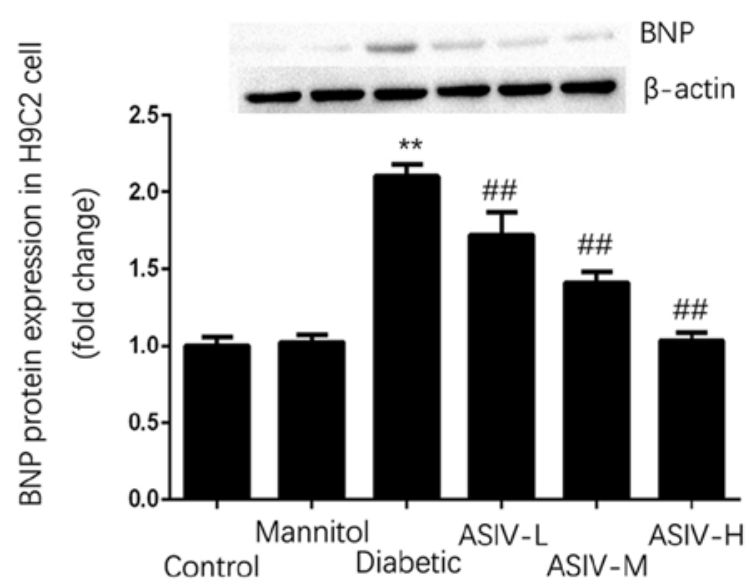

Control Diabetic ASIV-L ASIV-M ASIV-H
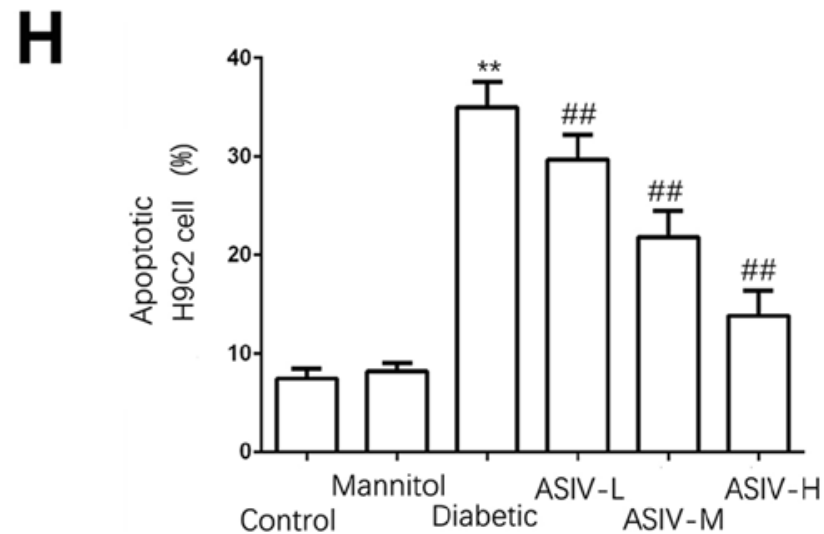

Figure 3. ASIV decreases the damage of myocardial tissue of diabetic rats and H9C2 cells in high-glucose environment. Protein expression of ANP in (A) the myocardium and (B) H9C2 cells. The protein expression of BNP in (C) the myocardium and (D) H9C2 cells. (E) Myocardial apoptosis assayed by TUNEL staining. TUNEL-positive cells were manifested as a marked appearance of dark brown apoptotic cell nuclei, and (F) the percentage of apoptotic cells in myocardial tissue. (G) The detection of apoptosis in H9C2 cells by AnnexinV-FITC and PI double staining, and (H) the percentage of apoptotic H9C2 cells. 

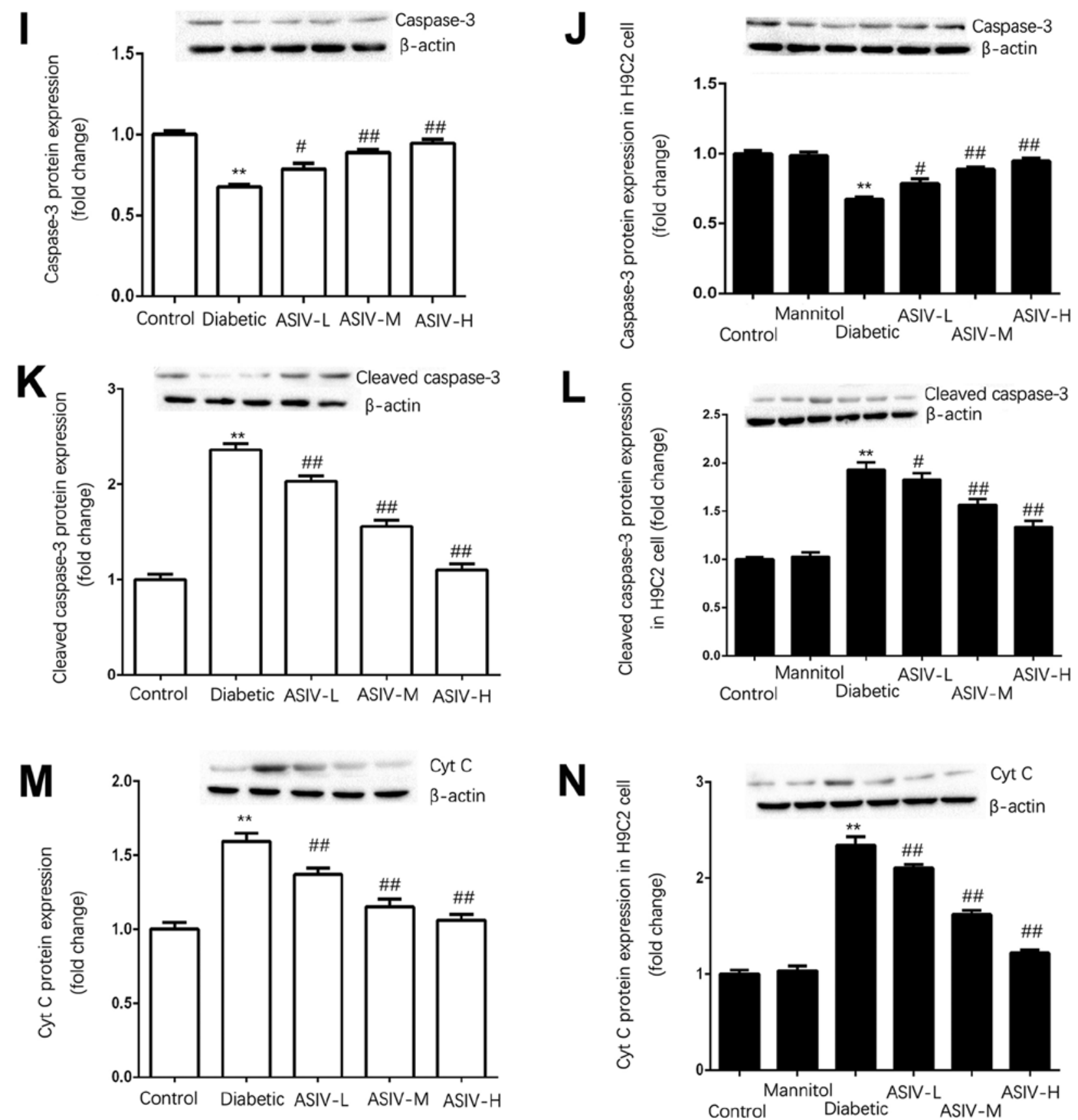

Figure 3. Continued. The protein expression of (I) caspase-3, (J) cleaved caspase-3 and (K) cytoplasmic Cyt C in the myocardium, and (L) caspase-3, (M) cleaved caspase-3 and $(\mathrm{N})$ cytoplasmic Cyt $\mathrm{C}$ in $\mathrm{H} 9 \mathrm{C} 2$ cells. The data are expressed as the means $\pm \mathrm{SD}$. $\mathrm{n}=4 .{ }^{* *} \mathrm{P}<0.01$ vs. control group. ${ }^{*} \mathrm{P}<0.05$ and ${ }^{\# \#} \mathrm{P}<0.01$ vs. diabetic group. The black arrows indicate the nucleus of apoptotic cardiomyocytes. ANP, atrial natriuretic peptide; ASIV, astragaloside IV; L, low; $\mathrm{M}$, mid; $\mathrm{H}$, high; BNP, brain natriuretic peptide; PI, propidium iodide; Cyt C, cytochrome $c$.

TUNEL staining and FITC/PI double staining were used to detect the apoptosis of the myocardial tissue and H9C2 cells, respectively (Fig. 3E-H). In the myocardial tissue and $\mathrm{H} 9 \mathrm{C} 2$ cells, the apoptotic rate of the control group was $<10 \%$. Apoptosis was significantly increased in the diabetic group and HG group compared with the control in tissue and cells. The apoptotic rate was dose-dependently decreased by ASIV compared with the diabetic model groups.

The expression of caspase-3, cleaved caspase-3 and cytoplasmic Cyt C (Fig. 3I-N), revealed that the expression of Cyt $\mathrm{C}$ in the cytoplasm and cleaved caspase- 3 was significantly increased and that caspase- 3 was decreased in the model group, whereas the expression of Cyt $\mathrm{C}$ and cleaved caspase-3 in the control group was not significantly different from that in the mannitol group; this excluded the influence of osmotic pressure on the results. Following ASIV administration, the protein expression of Cyt $\mathrm{C}$ and cleaved caspase-3 were decreased both in heart tissues and $\mathrm{H} 9 \mathrm{C} 2$ cells, whereas caspase-3 was significantly increased. The results demonstrated that ASIV can downregulate the increased expression of $\mathrm{Cyt} \mathrm{C}$ and cleaved caspase- 3 induced by the diabetes model.

ASIV can regulate the expression of energy metabolism-associated signals and the level of energy metabolism in myocardial 

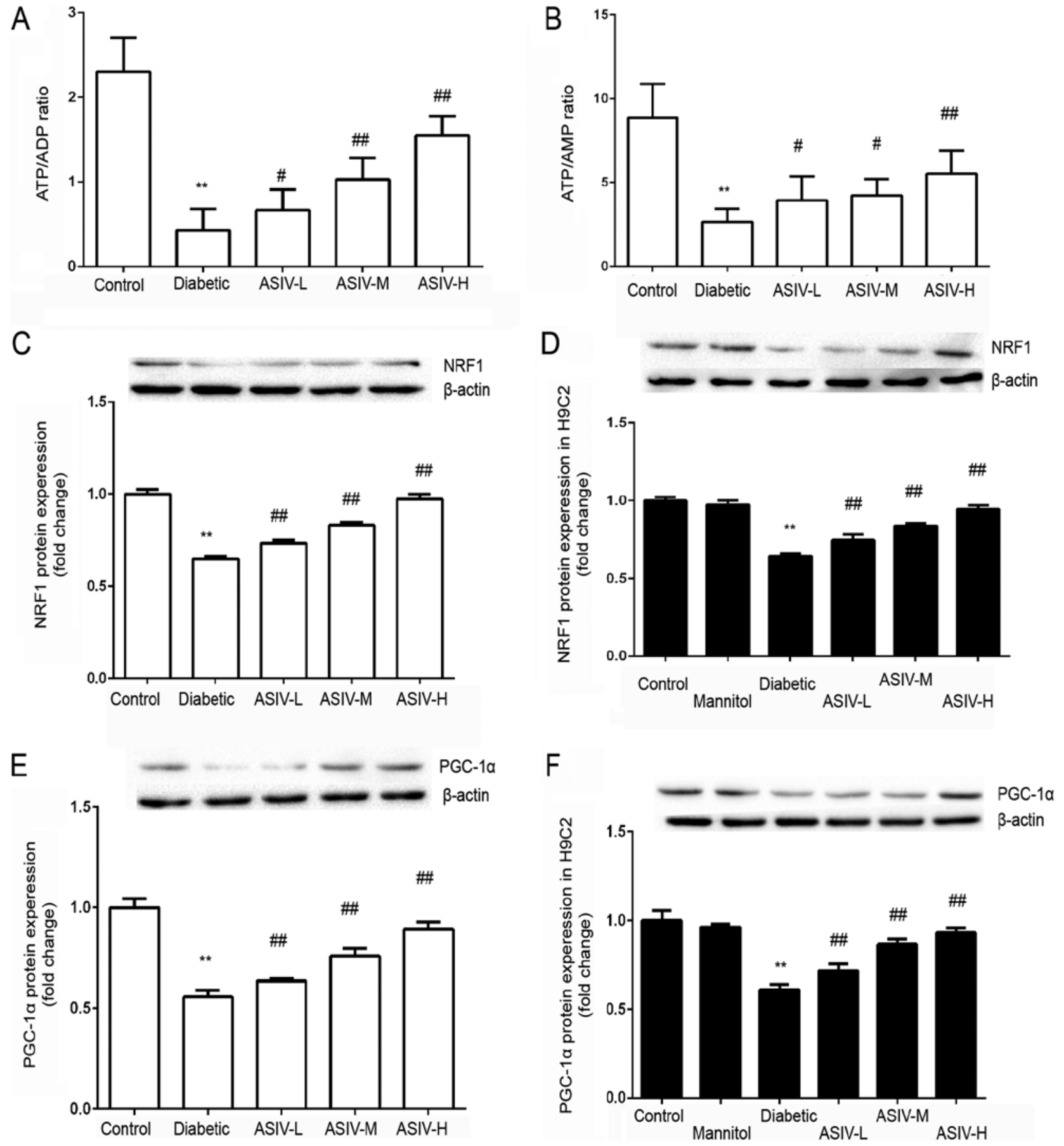

Figure 4. ASIV regulates the expression of energy metabolism associated signals and the level of energy metabolism in myocardial tissue and H9C2 cells. Ratio of (A) ATP/ADP and (B) ATP/AMP in H9C2 cells. $n=8$. The protein expression of NRF1 in (C) the myocardium and (D) H9C2 cells. $n=4$. The protein expression of PGC-1 $\alpha$ in (E) the myocardium and (F) H9C2 cells. $n=4$. The data are expressed as the mean $\pm \mathrm{SD}$. ${ }^{* *} \mathrm{P}<0.01$ vs. control group. ${ }^{*} \mathrm{P}<0.05$ and

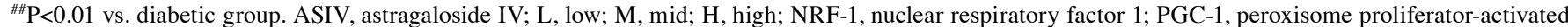
receptor $\lambda$ coactivator $1-\alpha$.

tissue and $H 9 C 2$ cells. The ATP/ADP and ATP/AMP ratios of each group were calculated. The results show that, compared with that of the control group, the ratios were significantly decreased in the diabetic group. These ratios were increased by the different doses of ASIV (Fig. 4A and B).

Compared with that in the control group and the model (diabetic and HG) groups, the expression of PGC-1 $\alpha$ and NRF1 was significantly decreased in the model group (Fig. 4C-F). Following ASIV administration, the expression of PGC-1 $\alpha$ and NRF1 was increased, and the expression increased with increasing ASIV concentration. This indicated that ASIV may improve myocardial energy metabolism abnormalities caused by diabetes. By detecting the mRNA expression of PGC- $1 \alpha$ and NRF1 in the H9C2 cells, under high-glucose conditions, the influence of osmotic pressure was determined and obtained the same result as that of histone expression, fully demonstrating the function of ASIV in improving myocardial energy metabolism. 


\section{Discussion}

The main cause of morbidity and mortality in diabetic patients is cardiovascular complications, which accounts for $80 \%$ of mortalities in diabetic patients (23). The most notable cardiovascular complication is diabetic cardiomyopathy, which increases the risk of future heart failure 5-fold (24-28). Diabetes has adverse effects on the different cell types of the heart, including endothelial cells $(29,30)$, fibroblasts and myocardial cells (31), and the damage to myocardial cells may be the main cause of heart failure (31-35). With the development of diabetes mellitus, the heart experiences cardiac hypertrophy, decreased compliance, decreased contractility and cell stability damage, leading to cardiac dysfunction (36). The number of apoptotic and necrotic cardiomyocytes began to increase. When the normal physiological structure was destroyed to a certain extent, compensatory adjustment can no longer compensate for myocardial damage, ultimately leading to heart failure (37).

Damage to the myocardium was mainly manifested by myocardial hypertrophy and apoptosis. ASIV, as one of the main active components in Astragalus, is a purified small-molecule saponin. According to existing studies, ASIV has anti-inflammatory and antioxidant effects. In previous studies, ASIV demonstrated efficacious inhibitory effect on apoptosis in vascular endothelial cells and to regulate the energy metabolism of vascular endothelial cells $(15-20,38,39)$. Additionally, in other experiments, it was found that its anti-inflammatory and antioxidant effects can improve the hypertrophy and apoptosis of the myocardium due to inflammation. Therefore, a diabetes model and the administration of ASIV was conducted, in order to study the effect and mechanism of ASIV in diabetic cardiomyopathy.

For the choice of index of hypertrophy, ANP and BNP were measured. Of course, there are other subtypes of natriuretic peptide, especially C-type natriuretic peptide (CNP) and N-terminal-proBNP (NT-proBNP) (40). CNP is involved in the regulation of myocardial changes in myocardial infarction, ischemia-reperfusion and heart failure, but its expression level was also affected by blood pressure and vascular endothelial cells, which cannot specifically respond to myocardial changes. Although NT-proBNP has no biological activity, it has a longer half-life and is more stable than BNP, and has been proposed as a biomarker of heart failure by the American Society of Clinical Chemistry (40-42). However, NT-proBNP could not reflect the changes in cardiac myocytes. ANP and BNP, as important biological signals of myocardial hypertrophy, are mainly synthesized in the atrium and left ventricle respectively, and in addition to indexes such as heart weight index (HWI) and left ventricular weight index (LVWI), ANP and BNP correspond to increased wall tension (43-46). The plasma level of polypeptides was shown to be positively correlated with the pressure caused by the filling of the heart, making it a good marker of LV dysfunction and LV wall stress abnormalities. There was a 23- to 85-fold increase in BNP, particularly in tissues with hypertrophy, compared with that in the control group. Apoptosis is regulated by a series of enzyme-linked reactions, mainly including two signal transduction pathways. One is the extrinsic pathway, that is, the death receptor pathway, which is under the stimulation of external factors; extracellular signal are transmitted to the intracellular caspase-protease promoter enzyme (47). The other is the intrinsic pathway, that is, the mitochondrial pathway (48). Caspase-3, the core protease in the apoptosis signal transduction pathway and an important executive enzyme for apoptosis, acts downstream in both the external pathway and the internal pathway $(48,49)$. The protein expression of caspase-3, as a downstream regulator in the death receptor pathway and mitochondrial apoptosis pathway, directly affects the apoptosis of myocardial cells. After Cyt C is transported from the mitochondria to the cytoplasm, it regulates the activity of the downstream caspase- 3 enzyme and promotes the apoptosis of myocardial cells $(50,51)$.

In the present study, STZ was used to establish a diabetes model, and gradient ASIV was used to treat diabetes model animals. The myocardial injury indicators were examined, including myocardial hypertrophy and myocardial apoptosis. HWI, LVWI, ANP and BNP increased, and myocardial hypertrophy occurred in the model group. The expression of cytoplasmic Cyt $\mathrm{C}$ and cleaved caspase-3 was increased, and these data combined with the flow cytometry results and TUNEL staining results suggest that apoptosis was increased in the model group. Following ASIV treatment, the indexes of myocardial hypertrophy and myocardial apoptosis recovered to varying degrees. The same results were obtained in H9C2 cells, indicating that ASIV has a therapeutic effect on diabetic myocardial injury.

How does ASIV improve myocardial injury in diabetes? Thus far, previous studies have established the correlation between diabetic complications and mitochondrial dysfunction in multiple tissues (52-54), but the potential mechanism of mitochondrial dysfunction in DCM was not clarified. Normally, the heart relies on persistent aerobic metabolism to maintain the ATP supply and redox balance for optimal systolic function. More than $90 \%$ of heart metabolism is aerobic $(55,56)$. Hyperglycemia is a major driver of mitochondrial dysfunction in diabetic myocardium (57-59). Due to the high-energy demand of the heart and the relief of myocardial injury in diabetic cardiomyopathy following abnormal energy metabolism is corrected, mitochondrial dysfunction was considered to be the cause of DCM (60-62). Studies using noninvasive 31p nuclear magnetic resonance imaging found that left ventricular hypertrophy and contractile inhibition are associated with decreased ATP levels, indicating that mitochondrial function was impaired under high-glucose conditions $(63,64)$. PGC-1 $\alpha$ is the most important coactivator in mitochondrial synthesis and energy metabolism and has a crucial role in regulating mitochondrial biosynthesis. $\mathrm{PGC}-1 \alpha$ regulates mitochondrial biosynthesis by activating downstream transcription factors, such as nuclear respiration factor (NRF). NRF is the most important transcription factor in the regulation of mitochondrial biosynthesis (65). The nuclear transcription factor NRF1 regulates the transcription, translation and replication of mitochondrial DNA and the expression of various proteins, including enzymes in the respiratory chain that promote oxidative energy supply, in the mitochondria.

ASIV was postulated to affect mitochondrial synthesis by regulating PGC-1 $\alpha$ and NRF1, thereby improving mitochondrial energy metabolism and decreasing the myocardial damage caused by diabetic cardiomyopathy. The expression of PGC-1 $\alpha$ and NRF1 protein in myocardial tissues, and the 
mRNA expression of PGC-1 $\alpha$ and NRF1 in H9C2 cells were detected in each group. ASIV upregulated the expression of PGC- $1 \alpha$ and NRF1, and the levels of ATP and ADP in H9C2 cells were also detected. The results showed that ASIV improved the energy metabolism level.

Based on the findings of the present study, it can be concluded that ASIV can decrease the damage of myocardial hypertrophy and apoptosis by improving mitochondrial energy metabolism via regulating PGC- $1 \alpha$ and NRF1 in diabetic cardiomyopathy. Moreover, the experimental results showed that ASIV had a hypoglycemic effect, but the underlying mechanism was still unclear. In addition, based on previous studies, ASIV also has a protective and therapeutic role in ischemia-reperfusion and oxidative stress on the heart; therefore, the protective effect of ASIV on diabetes mellitus on cardiomyopathy is not specific. Thus, ASIV has a broad protective effect on the heart.

In conclusion, ASIV can ameliorate myocardial mitochondrial energy metabolism abnormalities induced by diabetes mellitus via regulating the expression of PGC-1 $\alpha$ and NRF1. Improving abnormal energy metabolism in mitochondria can inhibit apoptosis and myocardial hypertrophy, thereby reducing myocardial injury. Thus, ASIV has the potential to alleviate myocardial damage induced by diabetes, by improving energy metabolism.

\section{Acknowledgements}

The authors would like to thank the laboratory teachers Dr Meili Lu, Dr Yang Liu, Dr Tairan Sun, Mr. Cong Li, Mr. Kun Zhao, Miss Tong Zhang, Miss Xiaoyao Zhang and Miss Yang Sun (Key Laboratory of Cardiovascular and Cerebrovascular Drug Researh of Liaoning Province, Junzhou Medical University) for providing technical support in the course of the experiment.

\section{Funding}

This work was supported by the National Nature Science Foundation of China (grant nos. 81673632 and 81703793) and LiaoNing Revitalization Talents Program (grant no. XLYC1802017).

\section{Availability of data and materials}

The datasets used and/or analyzed during the current study are available from the corresponding author on reasonable request

\section{Authors' contributions}

HW and ZZ designed the whole experiment, and contributed to the writing and modification of the article. ZZ, HZ, YZ and JW were responsible for the collection of data. $\mathrm{ZZ}$ and $\mathrm{HZ}$ performed the analysis and interpretaion of the data. All authors read and approved the final manuscript.

\section{Ethics approval and consent to participate}

All experiments and procedures were approved by the Medical Ethics Committee of Jinzhou Medical University (approval No. LNMU-2016-121). The raising of animals and the use of drugs are in accordance with the guidelines for the Care and use of Experimental Animals (8th Edition, Press of the National Academy of Sciences).

\section{Patient consent for publication}

Not applicable.

\section{Competing interests}

The authors declare that they have no competing interests.

\section{References}

1. Shaw JE, Sicree RA and Zimmet PZ: Global estimates of the prevalence of diabetes for 2010 and 2030. Diabetes Res Clin Pract 87: 4-14, 2010.

2. Lee CD, Folsom AR, Pankow JS and Brancati FL; Atherosclerosis Risk in Communities (ARIC) Study Investigators: Cardiovascular events in diabetic and nondiabetic adults with or without history of myocardial infarction. Circulation 109: 855-860, 2004.

3. Knapp M, Tu X and Wu R: Vascular endothelial dysfunction, a major mediator in diabetic cardiomyopathy. Acta Pharmacol Sin 40: 1-8, 2019.

4. Escaned J, Colmenárez H, Ferrer MC, Gutiérrez M, Jiménez-Quevedo P, Hernández R, Alfonso $\mathrm{F}$, Bañuelos $\mathrm{C}$, Deisla LP, Zamorano JL and Macaya C: Diastolic dysfunction in diabetic patients assessed with Doppler echocardiography: Relationship with coronary atherosclerotic burden and microcirculatory impairment. Rev Esp Cardiol 62: 1395-1403, 2009.

5. Zheng X, Sun T and Wang X: Activation of type 2 cannabinoid receptors (CB2R) promotes fatty acid oxidation through the SIRT1/PGC-1 $\alpha$ pathway. Biochem Biophys Res Commun 436: 377-381, 2013.

6. Sun X, Chen RC, Yang ZH, Sun GB, Wang M, Ma XJ, Yang LJ and Sun XB: Taxifolin prevents diabetic cardiomyopathy in vivo and in vitro by inhibition of oxidative stress and cell apoptosis. Food Chem Toxicol 63: 221-232, 2014.

7. Rowe GC, Jiang A and Arany Z: PGC-1 coactivators in cardiac development and disease. Circ Res 107: 825-838, 2010.

8. Arany Z, He H, Lin J, Hoyer K, Handschin C, Toka O, Ahmad F, Matsui T, Chin S, Wu PH, et al: Transcriptional coactivator PGC-1 alpha controls the energy state and contractile function of cardiac muscle. Cell Metab 1: 259-271, 2005.

9. Sebastiani M, Giordano C, Nediani C, Travaglini C, Borchi E, Zani M, Feccia M, Mancini M, Petrozza V, Cossarizza A, et al: Induction of mitochondrial biogenesis is a maladaptive mechanism in mitochondrial cardiomyopathies. J Am Coll Cardiol 50: 1362-1369, 2007.

10. Koh JH, Hancock CR, Terada S, Higashida K, Holloszy JO and Han DH: PPAR $\beta$ is essential for maintaining normal levels of PGC-1 $\alpha$ and mitochondria and for the increase in muscle mitochondria induced by exercise. Cell Metab 25: 1176-1185.e5, 2017.

11. Wu Z, Puigserver P, Andersson U, Zhang C, Adelmant G, Mootha V, Troy A, Cinti S, Lowell B, Scarpulla RC and Spiegelman BM: Mechanisms controlling mitochondrial biogenesis and respiration through the thermogenic coactivator PGC-1. Cell 98: 115-124, 1999.

12. Nakamura H, Matoba S, Iwai-Kanai E, Kimata M, Hoshino A, Nakaoka M, Katamura M, Okawa Y, Ariyoshi M, Mita Y, et al: p53 promotes cardiac dysfunction in diabetic mellitus caused by excessive mitochondrial respiration-mediated reactive oxygen species generation and lipid accumulation. Circ Heart Fail 5: 106-115, 2012.

13. Anderson EJ, Rodriguez E, Anderson CA, Thayne K, Chitwood WR and Kypson AP: Increased propensity for cell death in diabetic human heart is mediated by mitochondrial-dependent pathways. Am J Physiol Heart Circ Physiol 300: H118-H124, 2011.

14. Mei M, Tang F, Lu M, He X, Wang H, Hou X, Hu J, Xu C and Han R: Astragaloside IV attenuates apoptosis of hypertrophic cardiomyocyte through inhibiting oxidative stress and calpain-1 activation. Environ Toxicol Pharmacol 40: 764-773, 2015. 
15. Zhang S, Tang F, Yang Y, Lu M, Luan A, Zhang J, Yang $J$ and Wang $H$ : Astragaloside IV protects against isoproterenol-induced cardiac hypertrophy by regulating NF- $\kappa \mathrm{B} / \mathrm{PGC}-1 \alpha$ signaling mediated energy biosynthesis. PLoS One 10: e0118759, 2015.

16. Jia G, Leng B, Wang H and Dai H: Inhibition of cardiotrophin-1 overexpression is involved in the anti-fibrotic effect of Astrogaloside IV. Mol Med Rep 16: 8365-8370, 2017.

17. Dai H, Jia G, Lu M, Liang $\mathrm{C}$, Wang $\mathrm{Y}$ and Wang $\mathrm{H}$ : Astragaloside IV inhibits isoprenaline-induced cardiac fibrosis by targeting the reactive oxygen species/mitogen-activated protein kinase signaling axis. Mol Med Rep 15: 1765-1770, 2017.

18. Xu C, Tang F, Lu M, Yang J, Han R, Mei M, Hu J and Wang H: Pretreatment with Astragaloside IV protects human umbilical vein endothelial cells from hydrogen peroxide induced oxidative stress and cell dysfunction via inhibiting eNOS uncoupling and NADPH oxidase-ROS-NF- $\kappa$ B pathway. Can J Physiol Pharmacol 94: 1132-1140, 2016.

19. Xu C, Tang F, Lu M, Yang J, Han R, Mei M, Hu J, Zhou M and Wang H: Astragaloside IV improves the isoproterenol-induced vascular dysfunction via attenuating eNOS uncoupling-mediated oxidative stress and inhibiting ROS-NF- $\mathrm{B}$ B pathways. Int Immunopharmacol 33: 119-127, 2016.

20. Lu M, Tang F, Zhang J, Luan A, Mei M, Xu C, Zhang S, Wang H and Maslov LN: Astragaloside IV attenuates injury caused by myocardial ischemia/reperfusion in rats via regulation of toll-like receptor $4 /$ nuclear factor- $\kappa \mathrm{B}$ signaling pathway. Phytother Res 29: 599-606, 2015.

21. Holly EN, Shimamoto A, Debold JF and Miczek KA: Sex differences in behavioral and neural cross-sensitization and escalated cocaine taking as a result of episodic social defeat stress in rats. Pyschopharmacology (Berl) 224: 179-188, 2012.

22. Walczak $M$ and Błasiak T: Midbrain dopaminergic neuron activity across alternating brain states of urethane anaesthetized rat. Eur J Neurosci 45: 1068-1077, 2017.

23. Seferović PM, Petrie MC, Filippatos GS, Anker SD, Rosano G, Bauersachs J, Paulus WJ, Komajda M, Cosentino F, de Boer RA, et al: Type 2 diabetes mellitus and heart failure: A position statement from the Heart Failure Association of the European Society of Cardiology. Eur J Heart Fail 20: 853-872, 2018.

24. Amos AF, McCarty DJ and Zimmet P: The rising global burden of diabetes and its complications: Estimates and projections to the year 2010. Diabet Med 14 (Suppl 5): S1-S85, 1997.

25. Dries DL, Sweitzer NK, Drazner MH, Stevenson LW and Gersh BJ: Prognostic impact of diabetes mellitus in patients with heart failure according to the etiology of left ventricular systolic dysfunction. J Am Coll Cardiol 38: 421-428, 2001.

26. Kannel WB, Hjortland M and Castelli WP: Role of diabetes in congestive heart failure: The framingham study. Am J Cardiol 34 29-34, 1974.

27. Alaei Faradonbeh N, Nikaeen F, Akbari M, Almasi N and Vakhshoori M: Cardiovascular disease risk prediction among Iranian patients with diabetes mellitus in Isfahan Province, Iran, in 2014, by using Framingham risk score, atherosclerotic cardiovascular disease risk score, and high-sensitive $\mathrm{C}$-reactive protein. ARYA Atheroscler 14: 163-168, 2018.

28. Tadic M and Cuspidi C: Obesity and heart failure with preserved ejection fraction: A paradox or something else? Heart Fail Rev 24: 379-385, 2019.

29. Icks A, Claessen H, Kirchberger I, Heier M, Peters A, Trentinaglia I, Giani G, von Scheidt W and Meisinger C: Mortality after first myocardial infarction in diabetic and non-diabetic people between 1985 and 2009. The MONICA/KORA registry. Eur J Epidemiol 29: 899-909, 2014.

30. Feuvray D: Diabetic cardiomyopathy. Arch Mal Coeur Vaiss 97: 261-265, 2004.

31. Boudina S and Abel ED: Diabetic cardiomyopathy revisited. Circulation 115: 3213-3223, 2007.

32. Fiordaliso F, Li B, Latini R, Sonnenblick EH, Anversa P, Leri A and Kajstura J: Myocyte death in streptozotocin-induced diabetes in rats in angiotensin II-dependent. Lab Invest 80: 513-527, 2000.

33. Di Carli MF, Janisse J, Grunberger G and Ager J: Role of chronic hyperglycemia in the pathogenesis of coronary microvascular dysfunction in diabetes. J Am Coll Cardiol 41: 1387-1393, 2003.

34. Riehle $\mathrm{C}$ and Bauersachs J: Of mice and men: Models and mechanisms of diabetic cardiomyopathy. Basic Res Cardiol 114: 2, 2018

35. Gilca GE, Stefanescu G, Badulescu O, Tanase DM, Bararu I and Ciocoiu M: Diabetic cardiomyopathy: Current approach and potential diagnostic and therapeutic targets. J Diabetes Res 2017: 1310265,2017
36. Voulgari C, Papadogiannis D and Tentolouris N: Diabetic cardiomyopathy: From the pathophysiology of the cardiac myocytes to current diagnosis and management strategies. Vasc Health Risk Manag 6: 883-903, 2010.

37. Lu M, Leng B, He X, Zhang Z, Wang H and Tang F: Calcium sensing receptor-related pathway contributes to cardiac injury and the mechanism of Astragaloside IV on cardioprotection. Front Pharmacol 9: 1163, 2018.

38. Guan FY, Yang SJ, Liu J and Yang SR: Effect of astragaloside IV against rat myocardial cell apoptosis induced by oxidative stress via mitochondrial ATP-sensitive potassium channels. Mol Med Rep 12: 371-376, 2015.

39. Kakoullis L, Giannopoulou E, Papachristodoulou E, Pantzaris ND, Karamouzos V, Kounis NG, Koniari I and Velissaris D: The utility of brain natriuretic peptides in septic shock as markers for mortality and cardiac dysfunction: A systematic review. Int J Clin Pract 73: e13374, 2019.

40. Pemberton CJ, Johnson ML, Yandle TG and Espiner EA: Deconvolution analysis of cardiac natriuretic peptides during acute volume overload. Hypertension 36: 355-359, 2000.

41. Tang WH, Francis GS, Morrow DA, Newby LK, Cannon CP, Jesse RL, Storrow AB and Christenson RH; NACB Committee: National academy of clinical biochemistry laboratory medicine practice guidelines: Clinical utilization of cardiac biomarker testing in heart failure. Clin Biochem 41: 210-221, 2008.

42. Li S, Xiao Z, Li L, Hu B, Zhou Z, Yi S, Luo J, Xie L, Nie B, Mo $L$ and Wang S: Establishment of normal reference values of NT-proBNP and its application in diagnosing acute heart failure in children with severe hand food and mouth disease. Medicine 97: e12218, 2018.

43. Candido R, Forbes JM, Thomas MC, Thallas V, Dean RG, Burns WC, Tikellis C, Ritchie RH, Twigg SM, Cooper ME and Burrell LM: A breaker of advanced glycation end products attenuates diabetes-induced myocardial structural changes. Circ Res 92: 785-792, 2003.

44. Connelly KA, Kelly DJ, Zhang Y, Prior DL, Martin J, Cox AJ, Thai K, Feneley MP, Tsoporis J, White KE, et al: Functional, structural and molecular aspects of diastolic heart failure in the diabetic (mRen-2)27 rat. Cardiovasc Res 76: 280-291, 2007.

45. Ritchie RH, Quinn JM, Cao AH, Drummond GR, Kaye DM, Favaloro JM, Proietto J and Delbridge LM: The antioxidant tempol inhibits cardiac hypertrophy in the insulin-resistant GLUT4-deficient mouse in vivo. J Mol Cell Cardiol 42: 1119-1128, 2007

46. Huynh K, McMullen JR, Julius TL, Tan JW, Love JE, Cemerlang N, Kiriazis H, Du XJ and Ritchie RH: Cardiac-specific IGF-1 receptor transgenic expression protects against cardiac fibrosis and diastolic dysfunction in a mouse model of diabetic cardiomyopathy. Diabetes 59: 1512-1520, 2010.

47. Das DK: Redox regulation of cardiomyocyte survival and death Antioxid Redox Signal 3: 23-37, 2001.

48. Webster KA, Graham RM and Bishopric NH: BNip3 and signal-specific programmed death in the heart. J Mol Cell Cardiol 38: 35-45, 2005

49. Das S, Khan N, Mukherjee S, Bagchi D, Gurusamy N, Swartz H and Das DK: Redox regulation of resveratrol-mediated switching of death signal into survival signal. Free Radic Biol Med 44: 82-90, 2008.

50. Lax NZ, Turnbull DM and Reeve AK: Mitochondrial mutations: Newly discovered players in neuronal degeneration. Neuroscientist 17: 645-658, 2011.

51. $\mathrm{Li} \mathrm{XD,} \mathrm{Li} \mathrm{XM,} \mathrm{Gu} \mathrm{JW} \mathrm{and} \mathrm{Sun} \mathrm{XC:} \mathrm{MiR-155} \mathrm{regulates}$ lymphoma cell proliferation and apoptosis through targeting SOCS3/JAK-STAT3 signaling pathway. Eur Rev Med Pharmacol Sci 21: 5153-5159, 2017.

52. Fetterman JL, Holbrook M, Westbrook DG, Brown JA, Feeley KP, Bretón-Romero R, Linder EA, Berk BD, Weisbrod RM, Widlansky ME, et al: Mitochondrial DNA damage and vascular function in patients with diabetes mellitus and atherosclerotic cardiovascular disease. Cardiovasc Diabetol 15: 53, 2016

53. Kalvala AK, Khan I, Gundu C and Kumar A: An overview on ATP dependent and independent proteases including an anterograde to retrograde control on mitochondrial function; Focus on diabetes and diabetic complications. Curr Pharm Des: Jul 18, 2019 (Epub ahead of print). doi: 10.2174/1381612825666190718153901.

54. Jin H, Zhu B, Liu X, Jin J and Zou H: Metabolic characterization of diabetic retinopathy: An ${ }^{1} \mathrm{H}-\mathrm{NMR}$-based metabolomic approach using human aqueous humor. J Pharm Biomed Anal 174: 414-421, 2019 
55. Rolfe DF and Brown GC: Cellular energy utilization and molecular origin of standard metabolic rate in mammals. Physiol Rev 77: 731-758, 1997.

56. Trost SU, Belke DD, Bluhm WF, Meyer M, Swanson E and Dillmann WH: Overexpression of the sarcoplasmic reticulum $\mathrm{Ca}(2+)$-ATPase improves myocardial contractility in diabetic cardiomyopathy. Diabetes 51: 1166-1171, 2002.

57. Camara AK, Bienengraeber M and Stowe DF: Mitochondrial approaches to protect against cardiac ischemia and reperfusion injury. Front Physiol 2: 13, 2011.

58. Chatham JC and Seymour AM: Cardiac carbohydrate metabolism in Zucker diabetic fatty rats. Cardiovasc Res 55: 104-112, 2002.

59. Dey S, DeMazumder D, Sidor A, Foster DB and O'Rourke B: Mitochondrial ROS drive sudden cardiac death and chronic proteome remodeling in heart failure. Circ Res 123: 356-371, 2018.

60. Wang Y, Gao P, Wei C, Li H, Zhang L, Zhao Y, Wu B, Tian Y, Zhang W, Wu L, et al: Calcium sensing receptor protects high glucose-induced energy metabolism disorder via blocking gp78-ubiquitin proteasome pathway. Cell Death Dis 8: e2799, 2017.

61. Ares-Carrasco S, Picatoste B, Camafeita E, Carrasco-Navarro S, Zubiri I, Ortiz A, Egido J, López JA, Tuñón J and Lorenzo O: Proteome changes in the myocardium of experimental chronic diabetes and hypertension: Role of PPAR $\alpha$ in the associated hypertrophy. J Proteomics 75: 1816-1829, 2012.
62. Tsushima K, Bugger H, Wende AR, Soto J, Jenson GA, Tor AR, McGlauflin R, Kenny HC, Zhang Y, Souvenir R, et al: Mitochondrial reactive oxygen species in lipotoxic hearts induce Post-translational modifications of AKAP121, DRP1, and OPA1 that promote mitochondrial fission. Circ Res 122: 58-73, 2018.

63. Knowlton AA, Chen L and Malik ZA: Heart failure and mitochondrial dysfunction: The role of mitochondrial fission/fusion abnormalities and new therapeutic strategies. J Cardiovasc Pharmacol 63: 196-206, 2014.

64. Song M, Mihara K, Chen Y, Scorrano L and Dorn GW II: Mitochondrial fission and fusion factors reciprocally orchestrate mitophagic culling in mouse hearts and cultured fibroblasts. Cell Metab 21: 273-286, 2015.

65. Recchia FA, McConnell PI, Bernstein RD, Vogel TR, Xu X and Hintze TH: Reduced nitric oxide production and altered myocardial metabolism during the decompensation of pacing-induced heart failure in the conscious dog. Circ Res 83: 969-979, 1998

This work is licensed under a Creative Commons Attribution-NonCommercial-NoDerivatives 4.0 International (CC BY-NC-ND 4.0) License. 